\title{
The influence of high-dose simvastatin and diltiazem on myocardium in rabbits: a haemodynamic study
}

\author{
Magdalena Jasińska, Jacek Owczarek, Daria Orszulak-Michalak
}

Department of Biopharmacy, Medical University of Lodz, Poland

Submitted: 25 August 2010

Accepted: 3 September 2010

Arch Med Sci 2011; 7, 3: 388-396

DOI: 10.5114/aoms.2011.23401

Copyright ( 2011 Termedia \& Banach

\begin{abstract}
Introduction: Simvastatin and diltiazem are often prescribed together for the treatment of hypercholesterolaemia in patients with hypertension and/or angina pectoris. However, diltiazem, a CYP3A inhibitor, is a well-recognized risk factor of skeletal muscle myopathy. It is not known whether such interaction also affects myocardial efficiency causing haemodynamic changes. The aim of the experiment was to establish the impact of simvastatin co-administered with diltiazem on the haemodynamic parameters after continuous infusion of dopamine.

Material and methods: The experiments were performed on 28 New Zealand white rabbits. The animals were divided into four groups receiving: $0.2 \% \mathrm{MC}-$ methylcellulose (control group); diltiazem; simvastatin; simvastatin + diltiazem, for 14 days ( $p o$ ). The following haemodynamic parameters were estimated: cardiac output index $(\mathrm{CI})$, heart rate (HR), systolic blood pressure (SBP), mean blood pressure (MBP), diastolic blood pressure (DBP) and total peripheral resistance index (TPRI). The registration of haemodynamic parameters was performed by the Doppler method and during the experiments the animals were anaesthetized with $\alpha$-chloralose $(75 \mathrm{mg} / \mathrm{kg} \mathrm{bw})$ and urethane $(500 \mathrm{mg} / \mathrm{kg} \mathrm{bw})$. Results: Dopamine did not cause a statistically significant increase in $\mathrm{Cl}$ in rabbits receiving simvastatin alone. Diltiazem significantly increased $\mathrm{Cl}$ if given simultaneously with simvastatin, which might suggest the improvement of cardiac efficiency resulting from such interaction.

Conclusions: The possibility of another mechanism of drug-drug interaction than the one based on CYP3A inhibition, and its impact on cardiac or skeletal muscle, might be considered.
\end{abstract}

Key words: statins, diltiazem, haemodynamic parameters, myopathy, myocardium, rabbits.

\section{Introduction}

3-hydroxy-3-methyl-glytaryl coenzyme A (HMG-CoA) reductase inhibitors (statins, HMGRI) have been proven to be extremely useful in the management of hypercholesterolaemia, especially with an elevated lowdensity lipoprotein cholesterol (LDL-C) level. Due to their pleiotropic activities, the long-term efficacy of statins for the primary and secondary [1-4] prevention of coronary heart disease is also well established. Until recently, the clinical trials have been performed to support the benefit of these agents for acute coronary syndromes [5].

Statins are well tolerated by most patients, but may produce a variety of muscle-related symptoms such as myopathy and rhabdomyolysis, accounting

\author{
Corresponding author: \\ Magdalena Jasińska MD, PhD \\ Department of Biopharmacy \\ Medical University of Lodz \\ 1 Muszyńskiego \\ Lodz, Poland \\ E-mail: \\ magdalena.jasinska@umed.lodz.pl
}


for $2 \%$ to $5 \%$ of all musculoskeletal effects. However, still little is known as to how statins produce muscle injury $[6,7]$. It has been shown that statin-related myotoxicity followed a dose-response curve. Thus, it is more likely to occur with higher serum statin concentrations. It may increase significantly when HMGRIs, including simvastatin, metabolized by the CYP3A isoenzyme, are prescribed concomitantly with other drugs inhibiting their metabolism via the CYP3A pathway (e.g. antifungal and immunosuppressive drugs, and macrolides). Co-administration of simvastatin and diltiazem or mibefradil has also been proven to increase the circulating simvastatin concentration [8-10].

Diltiazem, a calcium channel blocker (CCB), is a well-established anti-ischaemic agent, used especially in vasospastic angina pectoris (Guidelines on the Management of Stable Angina Pectoris), and quite frequently there is a need for its combined administration with statin. Simvastatin and diltiazem are often prescribed together for the treatment of hypercholesterolaemia in patients with hypertension and/or angina pectoris, and in such cases caution is recommended due to the risk of development of well-known musculoskeletal adverse effects. Moreover, in some cases the guidelines recommend such combination if the clinical benefit is likely to outweigh the increased risk of myopathy and rhabdomyolysis [11, 12]. There is little evidence as to whether such interaction similarly to skeletal muscle also affects cardiac muscle. Thus, taking into consideration the clinical importance of interactions resulting from the co-administration of CCBs and HMGRIs, the haemodynamic estimation of cardiac function, especially its efficiency, was planned. As a function of myocardial efficiency, one of its parameters - cardiac output resulting from a continuous infusion of a positive inotropic agent, dopamine - was assessed.

\section{Material and methods}

Drugs: simvastatin (Polfarmex, Poland series no. KY-SI-M20030102), diltiazem (Diltiazemi hydrochloridum, Polfarmex, Poland, series no. 010203), dopamine (Dopaminum hydrochloricum 1\%, Polfa Warszawa, Poland, series no. 01AV0203), $\alpha$-chloralose (Sigma, USA, series no. 120K2505), urethane (Sigma, USA, series no. 022K1248), lidocaine (Lidocaini hydrochloridum 2\%, Polfa Warszawa, Poland, series no. 14BT0403), methylcellulose (Fluka, Switzerland, series no RB 13425), natrium chloratum $0.9 \%$ inj. (Polfa Lublin, Poland, series no. 10602).

The experiments were performed on 28 outbred New Zealand white rabbits, both sexes, body weight 2.7-4.5 kg, fed on granulated mix LSK with free access to water. The animals were housed in standard cages, one animal per cage. The experimental procedures were carried out in accordance with the international guidelines for care and use of laboratory animals. All efforts were made to minimize animal suffering and reduce the number of animals used in the experiments. All the procedures in these experiments were approved by the Ethics Committee of the Medical University of Lodz, Poland ( $九 / B D / 131)$.

The animals were divided into four groups of rabbits, randomly allocated to each group: group I: $0.2 \%$ methylcellulose (MC), $1 \mathrm{ml} / \mathrm{kg}$ bw, po daily for 14 days $(n=9)$;

group II: diltiazem $5 \mathrm{mg} / \mathrm{kg}$ bw, po daily for 14 days $(n=6)$;

group III: simvastatin $50 \mathrm{mg} / \mathrm{kg}$ bw, po daily for 14 days $(n=6)$;

group IV: simvastatin $50 \mathrm{mg} / \mathrm{kg}$ bw + diltiazem $5 \mathrm{mg} / \mathrm{kg}$ bw, po daily for 14 days $(n=7)$.

All drugs, in doses used in the previous experiments in rabbits $[13,14]$, were administered by oral gavage, suspended in $0.2 \% \mathrm{MC}$.

\section{Haemodynamic studies}

The surgery was performed $24 \mathrm{~h}$ after the administration of the last drug dose. The rabbits were placed in a dorsal position on the operation table. The animals were anaesthetized with $\alpha$-chloralose (75 mg/kg bw) and urethane (500 mg/kg bw), administered into the marginal ear vein. Anaesthesia was maintained by additional bolus doses of urethane as needed. Lidocaine was used for local infiltration of the surgical sites. During the experimental procedures, the thoracic cavity was opened for further aortic flow measurement from the ascending aorta. Before thoracotomy, the trachea was intubated. Ventilator frequency was set at $30 \mathrm{bpm}$ and tidal volume at $10 \mathrm{ml} / \mathrm{kg}$ bw.

Dopamine, dissolved in $0.9 \% \mathrm{NaCl}$, was infused into the marginal ear vein, using a continuous infusion pump (sp100i syringe pump, WPI, England), at $10 \mu \mathrm{g} / \mathrm{kg}$ min bw. The infusion was begun after the stabilization period.

The registration of haemodynamic parameters in rabbits was performed using Hugo Sachs Electronik Haemodyn (Harvard Apparatus GmbH, March, Germany).

For the measurement of systolic, mean and diastolic arterial blood pressure (systolic blood pressure - SBP, mean blood pressure - MBP, diastolic blood pressure - DBP), a heparinized polyethylene catheter was placed into the dissected carotid artery and connected to an Isotec pressure transducer (HSE Harvard Apparatus, Germany).

After median sternotomy and pericardiotomy, an ultrasonic flow probe was placed around the ascending aorta and connected to an ultrasonic flow meter Transit Time Flowmeter TTFM Type 700 (HSE Harvard Apparatus and Transonic System Inc. 
USA), in order to measure aortic blood flow $\left(A F_{\text {max }}, A F_{\text {mean }}, A F_{\text {min }}\right)$. The $A F_{\text {mean }}$ values were taken as an index of cardiac output. The heart rate was registered from the catheter placed in the carotid artery. After surgery, the animals were killed by exsanguination while ventilation was continued.

All analogue signals were amplified and recorded on a computer via an A/D converter (HSE Haemodyn software for Microsoft Windows 95/98/NT) and evaluated according to the algorithms. For further statistical analysis, the mean from 3-minute periods was calculated as output values.

The following derivative haemodynamic parameters were calculated: cardiac output index $(\mathrm{Cl})$ and total peripheral resistance index (TPRI):

$-\mathrm{Cl}=\mathrm{CO} / \mathrm{BW}(\mathrm{ml} / \mathrm{min} \mathrm{kg}), \mathrm{CO}-$ cardiac output $(\mathrm{ml} / \mathrm{min}), \mathrm{BW}$ - body weight $(\mathrm{kg})$;

$-\mathrm{TPRI}=\mathrm{TPR} / \mathrm{BW}(\mathrm{mmHg} \mathrm{min} / \mathrm{ml} \mathrm{kg}), \mathrm{TPR}=$ MAP/CO (mmHg min/ml), MAP - mean arterial pressure $(\mathrm{mmHg})$.

\section{Statistic analysis}

The statistical analysis of haemodynamic parameters was carried out using the Statistica version 5.0 StatSoft program. The statistical evaluation was performed using the analysis of variance (ANOVA) and post-hoc comparisons were performed using the Duncan test. Normal distribution of a parameter was checked by means of the Kolmogorov-Smirnov test with the Lilliefors correction. The homogeneity of variance was tested by Levene's test. If data were not normally distributed or the values of variance were different, ANOVA with the Kruskal-Wallis and Mann-Whitney $U$ test was used.

All parameters were considered statistically significantly different if $p<0.05$.

\section{Results}

\section{Haemodynamic studies}

\section{Cardiac output index}

In the control group, the dopamine infusion caused a statistically significant increase in $\mathrm{Cl}$, observed from the $15^{\text {th }}$ min to the end of the experiment, maximally by $19 \%$. In rabbits receiving diltiazem, a significant increase in $\mathrm{Cl}$ was observed from the $9^{\text {th }}$ min to the end of the experiment, maximally by $37 \%$. The administration of simvastatin alone did not result in a statistically significant increase in $\mathrm{Cl}$ during the continuous infusion of dopamine. In rabbits receiving simvastatin simultaneously with diltiazem, the continuous infusion of dopamine caused a statistically significant increase in $\mathrm{Cl}$ values, maximally by $23 \%$, observed from the $6^{\text {th }}$ min after the infusion had been started (Figure 1).

The initial values of $\mathrm{Cl}$ noted in rabbits receiving simvastatin or diltiazem did not differ significantly from the values of $\mathrm{Cl}$ noted in the control group (Figure 1).

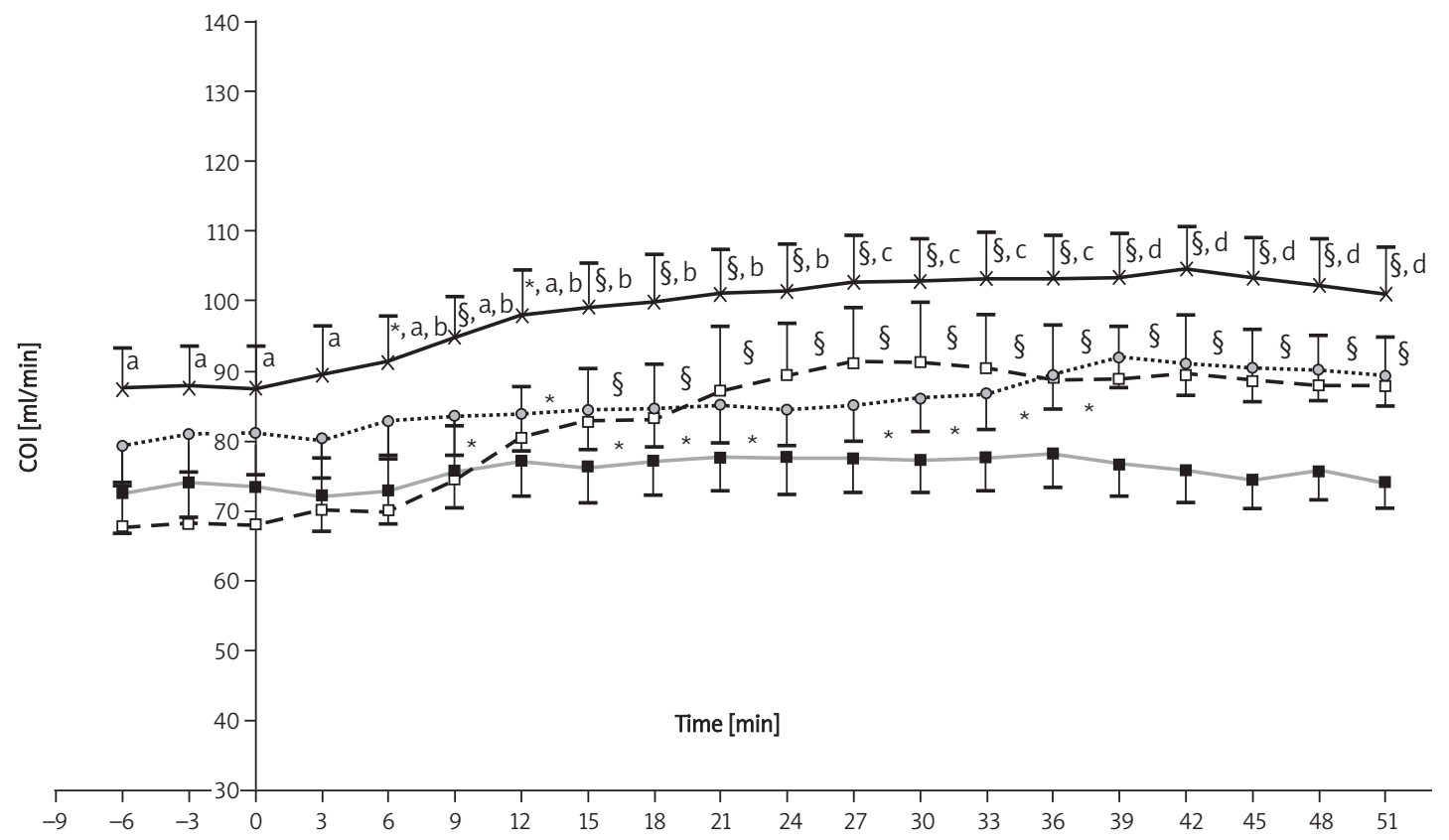

Figure 1. The influence of diltiazem $(\square)$, simvastatin $(\square)$, diltiazem + simvastatin $(x)$, in comparison to the control group (O), on cardiac output index (CI) in rabbits, during continuous infusion of dopamine, given simultaneously. Each value represents the mean \pm SEM. (a) $p<0.05$ in comparison to diltiazem alone, (b) $p<0.05$, (c) $p<0.01$, (d) $p<0.005$ in comparison to simvastatin alone, in comparison to diltiazem alone: $\left({ }^{\star}\right) p<0.05,(\$) p<0.005$ in comparison to the initial values ( $\left.0^{\prime}\right)$. $0^{\prime}$ - the initiation of dopamine infusion 
The combined administration of simvastatin with diltiazem caused a marked increase in the cardiac output index, observed from the $6^{\text {th }}$ min of the dopamine infusion as compared to simvastatin alone (Figure 1).

\section{Blood pressure}

Dopamine infusion did not influence systolic blood pressure values in the examined groups of rabbits. The values of SBP and MBP, observed after dopamine infusion had been started, did not differ significantly from the initial values.

The values of SBP observed in rabbits receiving diltiazem or simvastatin alone were significantly lower as compared to the control group. The combined administration of simvastatin and diltiazem caused a marked decrease in the values of SBP compared to the control group, both before and during the dopamine infusion (Figure 2).

In the control group, as well as in rabbits receiving diltiazem alone or simultaneously with simvastatin, dopamine did not cause any changes in MBP values. The simultaneous administration of simvastatin and diltiazem caused a marked decrease in MBP values, compared to the control group (Figure 3).

An insignificant influence of dopamine on DBP values was observed, except for the last $18 \mathrm{~min}$ in rabbits receiving simvastatin alone.

The values of DBP observed in rabbits receiving diltiazem were significantly lower as compared to the control group. The combined administration of simvastatin with diltiazem resulted in DBP reduction compared to the control group (Figure 4).

\section{Heart rate}

The administration of dopamine caused a marked reduction of the heart rate $(H R)$ values in groups receiving simvastatin alone, observed from the $9^{\text {th }}$ min of dopamine infusion to the end of the experiment. In rabbits receiving simvastatin with diltiazem, dopamine significantly slowed the rabbits' heart rate beginning from the $12^{\text {th }}$ min of its administration to the end of the experiment.

The administration of simvastatin alone or with diltiazem resulted in a decrease in $\mathrm{HR}$, compared to the control group. These changes were statistically significant after the dopamine infusion had started. In rabbits receiving simvastatin simultaneously with diltiazem, a marked decrease in HR compared to diltiazem alone was noted. These changes were statistically significant, beginning from the $18^{\text {th }} \mathrm{min}$ of the dopamine infusion (Figure 5).

\section{Total peripheral resistance index}

The infusion of dopamine caused a reduction in TPRI in the examined groups of rabbits. A significant decrease in TPRI in rabbits receiving diltiazem with simvastatin was observed after the $3^{\text {rd }}$ min of dopamine infusion. In the group receiving simvastatin alone, a marked reduction in TPRI was noted after the $30^{\text {th }}$ min of dopamine administration. In the control group, a significant decrease

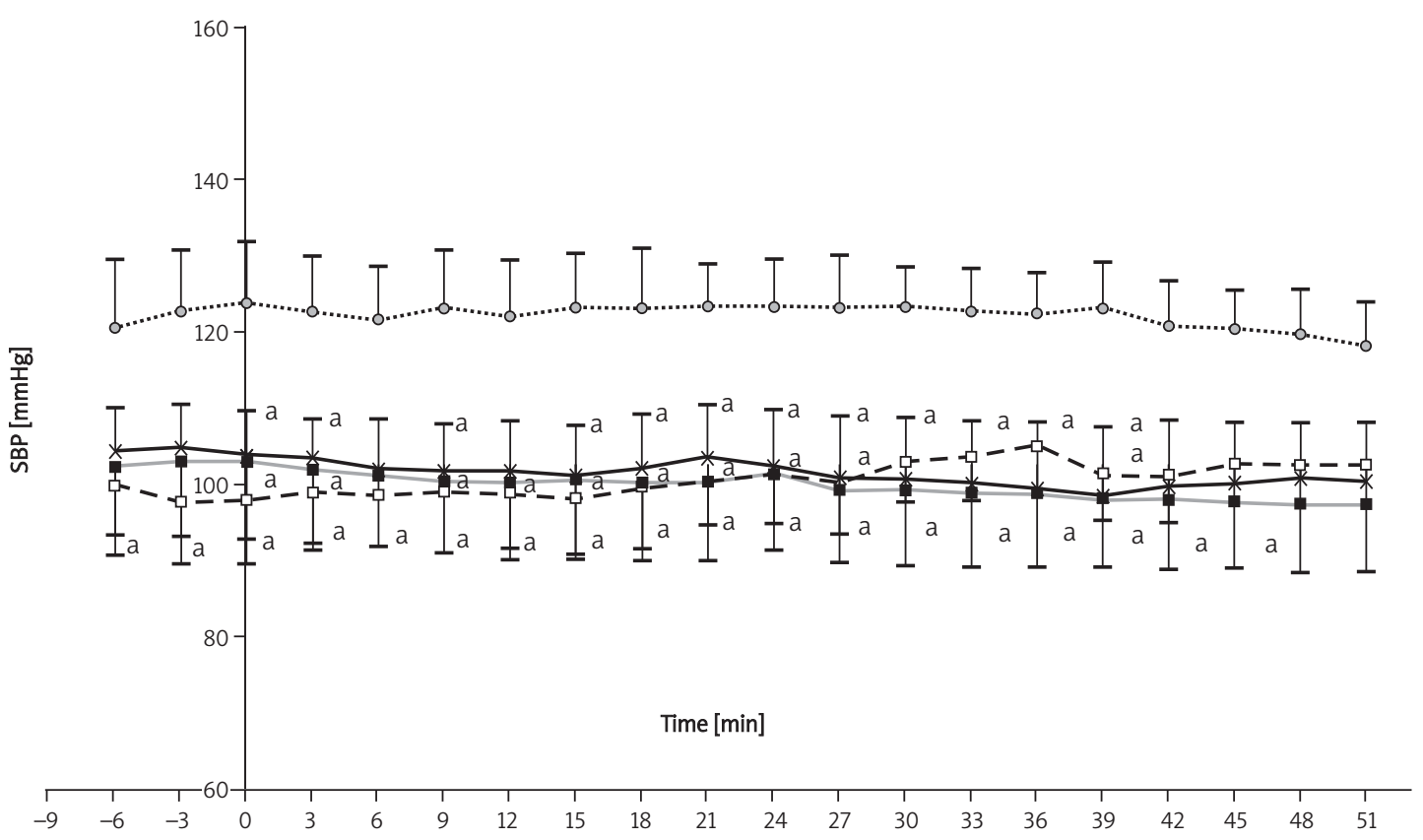

Figure 2. The influence of diltiazem $(\square)$, simvastatin $(\square)$, diltiazem + simvastatin $(x)$, in comparison to the control group (O), on systolic blood pressure (SBP) in rabbits, during continuous infusion of dopamine, given simultaneously. Each value represents the mean \pm SEM. (a) $p<0.05$ in comparison to the control group. $0^{\prime}-$ the initiation of dopamine infusion 
in TPRI was observed after the $27^{\text {th }}$ min of dopamine administration (Figure 6).

\section{Discussion}

Diltiazem is a recognized risk factor of statininduced myopathy of skeletal muscles. As a substrate and inhibitor of the human liver microsomal enzyme CYP3A, by which simvastatin is also metabolized, diltiazem significantly increases the mean peak serum concentration of simvastatin (by 3.6-fold) as well as the area under the serum concentration-time curve (by 5-fold) [15]. Pharmacokinetic interaction

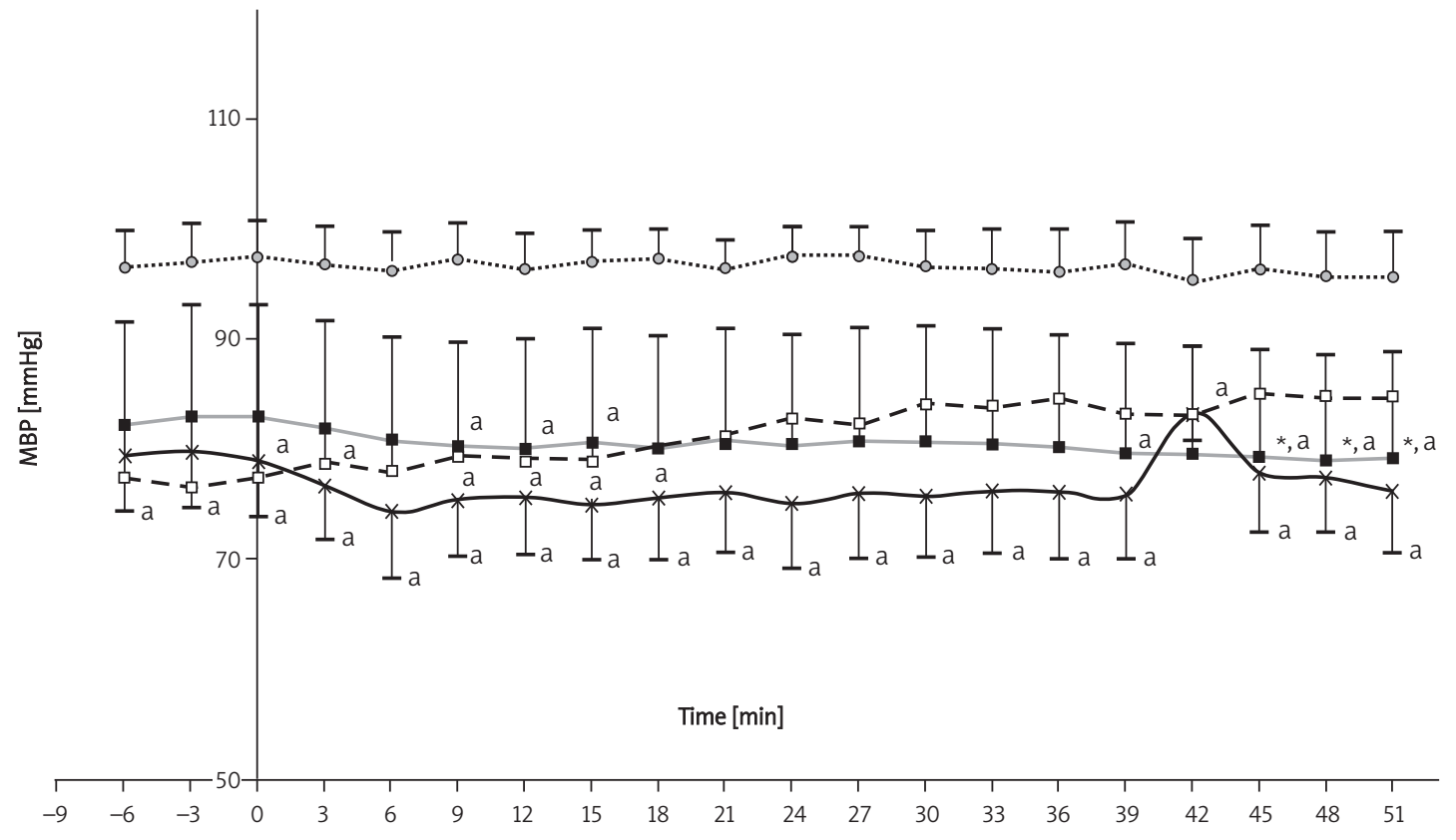

Figure 3. The influence of diltiazem $(\square)$, simvastatin $(\square)$, diltiazem + simvastatin $(x)$, in comparison to the control group (O), on mean blood pressure (MBP) in rabbits, during continuous infusion of dopamine, given simultaneously. Each value represents the mean \pm SEM. (a) $p<0.05$ in comparison to the control group, $\left({ }^{*}\right) p<0.05$ in comparison to the initial values $\left(0^{\prime}\right) .0^{\prime}-$ the initiation of dopamine infusion

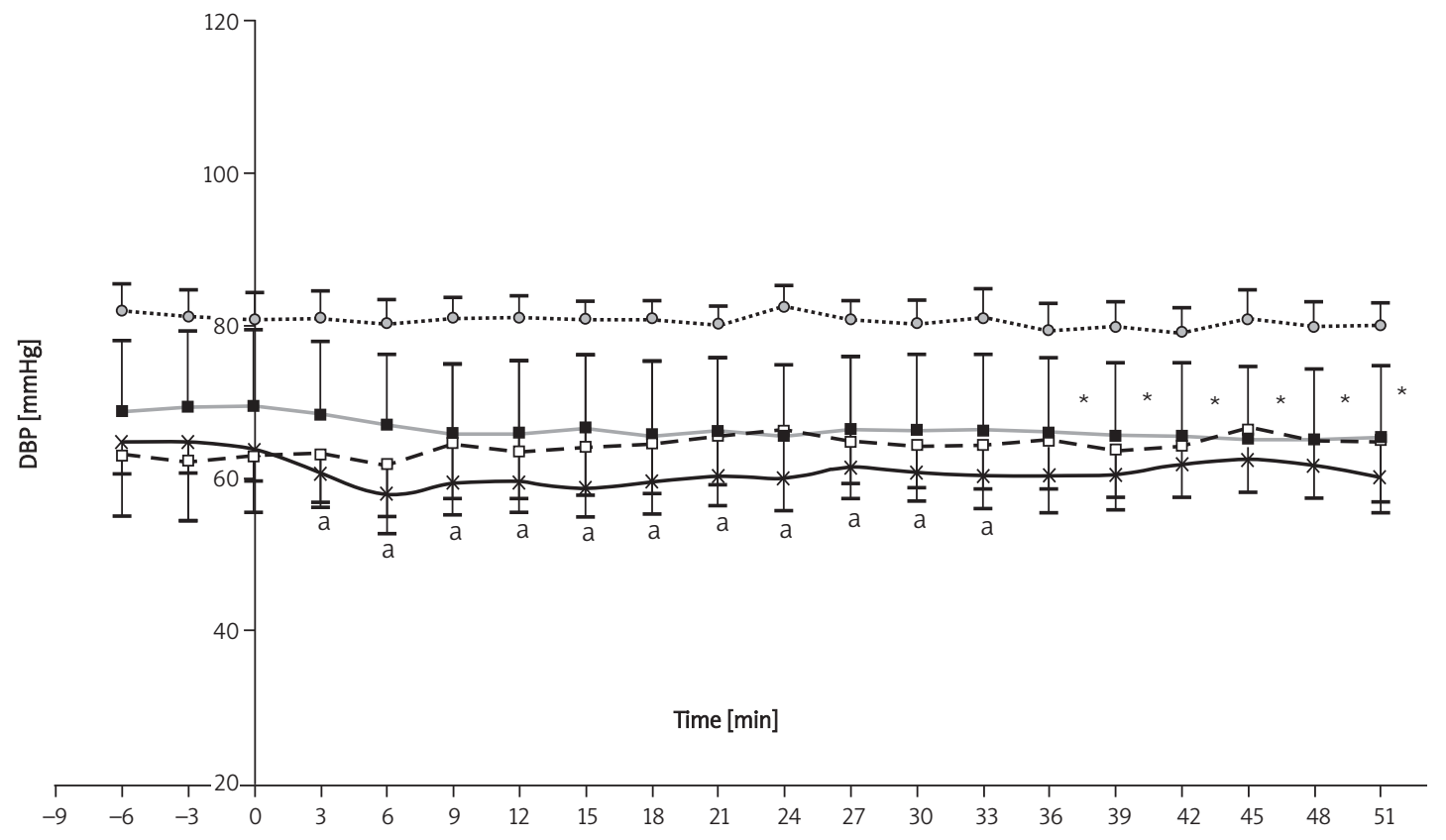

Figure 4. The influence of diltiazem $(\square)$, simvastatin $(\square)$, diltiazem + simvastatin (x), in comparison to the control group (O), on diastolic blood pressure (DBP) in rabbits, during continuous infusion of dopamine, given simultaneously. Each value represents the mean \pm SEM. (a) $p<0.05$ in comparison to the control group, $\left(^{*}\right) p<0.05$ in comparison to the initial values $\left(0^{\prime}\right)$. $0^{\prime}$ - the initiation of dopamine infusion 
between statin and diltiazem is known to enhance the pharmacodynamic effect of simvastatin and may lead to an increased risk of statin side effects, including myopathic symptoms. On the other hand, the magnitude of this interaction is smaller than observed with other CYP3A inhibitors such as itraconazole or mibefradil, a selective T-type calcium channel blocker inhibitor. Myopathy occurs in about $1 \%$ of patients taking simvastatin in higher doses with verapamil or with diltiazem, and considering the risk of such adverse musculoskeletal symptoms caution is recommended [16].

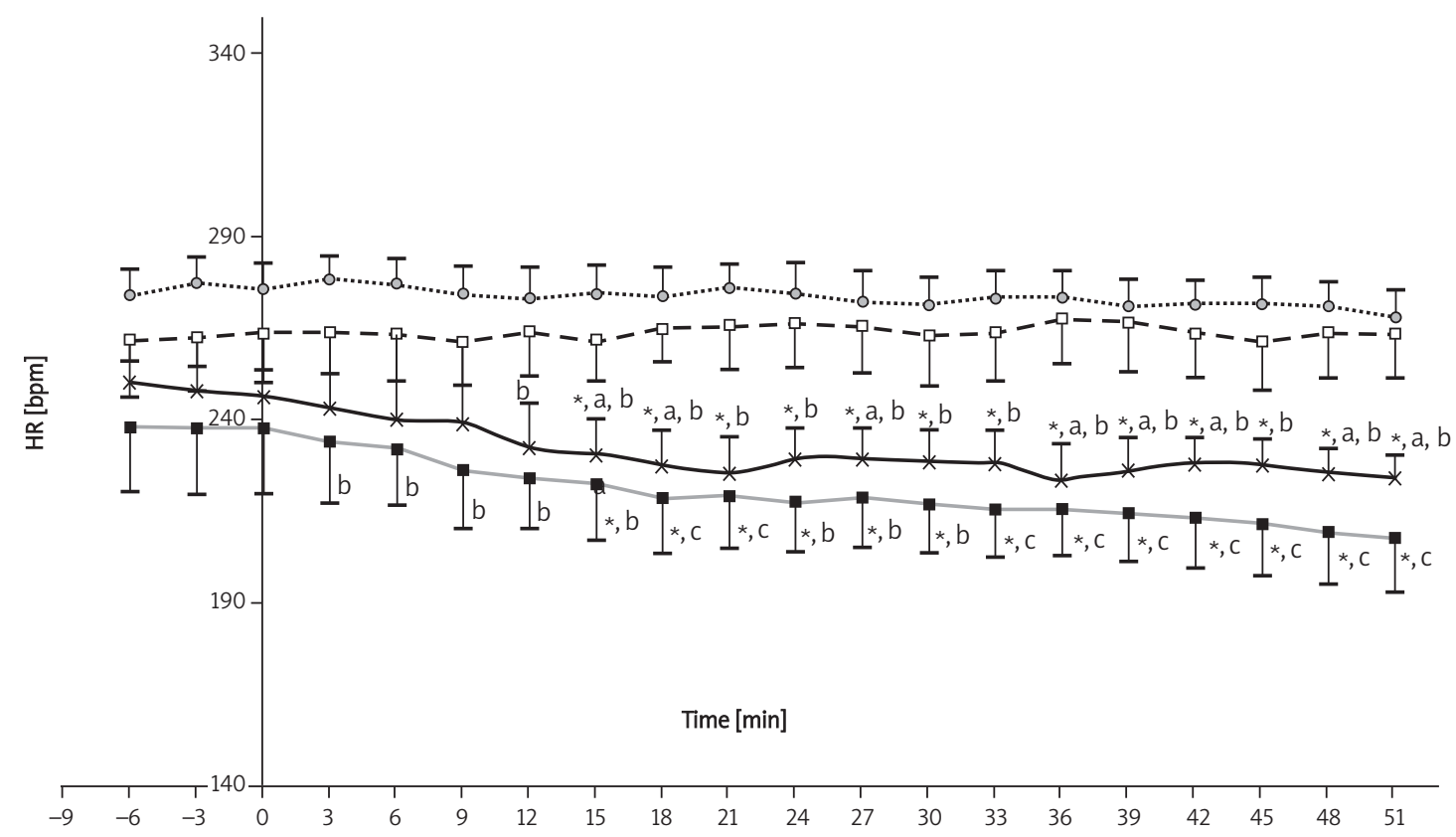

Figure 5. The influence of diltiazem $(\square)$, simvastatin $(\square)$, diltiazem + simvastatin $(x)$, in comparison to the control group (O), on heart rate (HR) in rabbits, during continuous infusion of dopamine, given simultaneously. Each value represents the mean \pm SEM. (a) $p<0.05$ in comparison to diltiazem alone, (b) $p<0.05$, (c) $p<0.005$ in comparison to the control group, $\left({ }^{*}\right) p<0.05$ in comparison to the initial values $\left(0^{\prime}\right) .0^{\prime}-$ the initiation of dopamine infusion

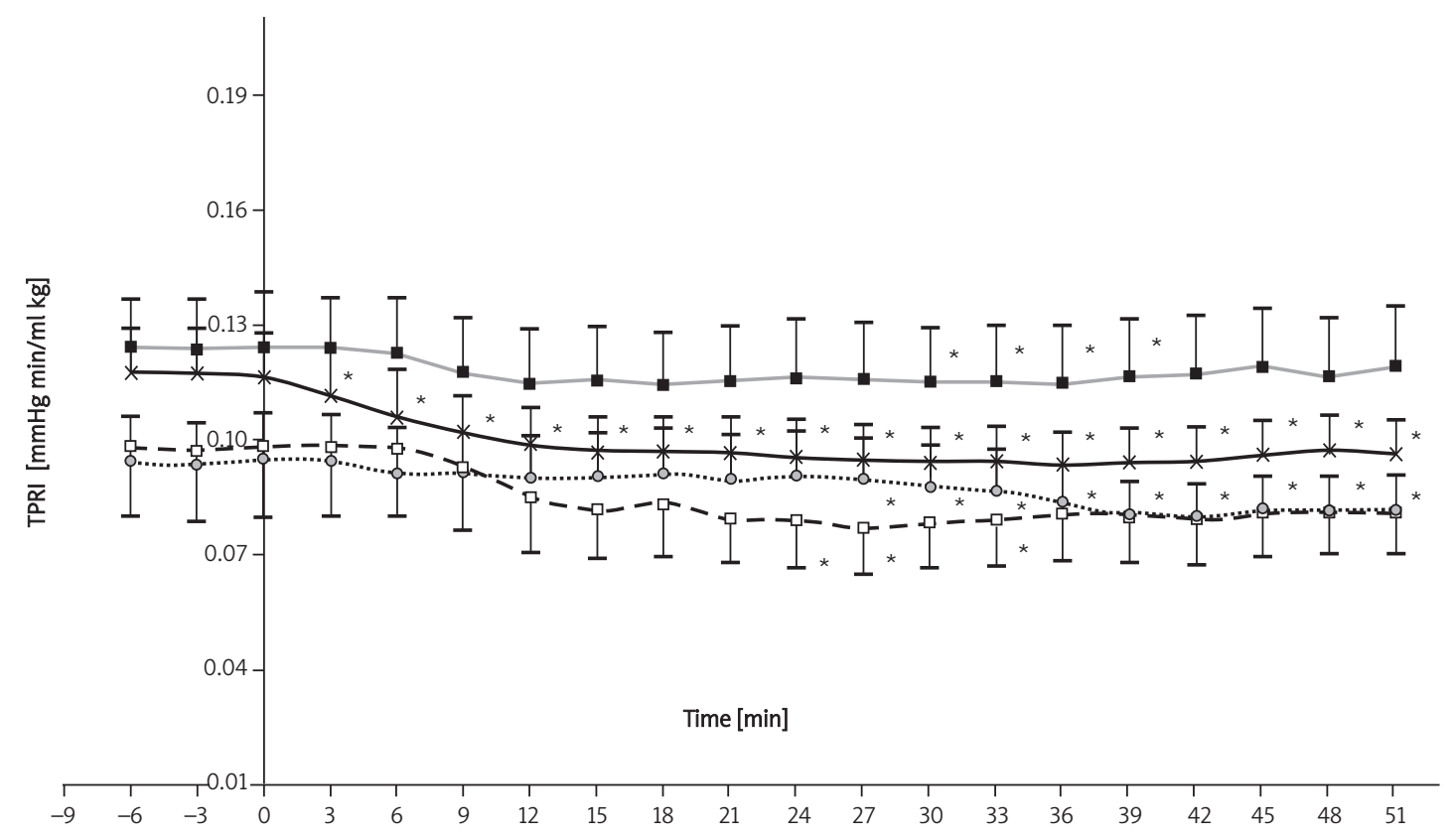

Figure 6. The influence of diltiazem $(\square)$, simvastatin $(\square)$, diltiazem + simvastatin $(x)$, in comparison to the control group (O), on total peripheral resistance index (TPRI) in rabbits, during continuous infusion of dopamine, given simultaneously. Each value represents the mean \pm SEM. $\left(^{*}\right) p<0.05$ in comparison to the initial values $\left(0^{\prime}\right)$. $0^{\prime}-$ the initiation of dopamine infusion 
Our findings indicate that diltiazem may improve cardiac efficacy in terms of statin administration. The simultaneous administration of simvastatin with diltiazem resulted in an increase in $\mathrm{Cl}$. The administration of simvastatin alone did not cause a significant reduction in the initial $\mathrm{Cl}$ values (i.e. before dopamine infusion); no response to dopamine inotropic activity was observed as opposed to the other groups. In contrast, we have shown that nifedipine significantly worsened the cardiac function if given simultaneously with simvastatin, suggesting that nifedipine may have a detrimental impact on statin therapy [17]. Diltiazem, but not nifedipine however, by inhibition of smooth muscle L-type calcium channels, decreases intracellular calcium concentration not only in smooth muscle cells but also in cardiomyocytes.

Calcium overload is one of the suggested factors responsible for skeletal muscle myopathy development. Nakahara et al. (1994) revealed that simvastatin and simvastatin acid elevated $\mathrm{Ca}^{2+}$ in L6 rat myoblasts [18]. Similarly, intracellular $\mathrm{Ca}^{2+}$ increase in cardiomyocytes was reported as well. If statin-induced $\mathrm{Ca}^{2+}$ elevation plays a role in development of myotoxicity, this could explain why concomitant administration of diltiazem and simvastatin improves cardiac efficiency compared to simvastatin alone. It is already known that diltiazem by inhibiting $\mathrm{Ca}^{2+}$ inflow to the ischaemic myocardium and preventing mitochondrial damage may have a positive influence on the performance of the damaged cardiac muscle, including diastolic dysfunction [19]. The cardioprotective properties of diltiazem were confirmed in another study by Wang et al. The authors suggested that diltiazem might restore cardiac output and improve renal function after haemorrhagic shock and crystalloid resuscitation [20].

The reduction of heart rate after simvastatin administration seems to be controversial considering its impact on $\mathrm{Ca}^{2+}$ uptake in cardiomyocytes. However, some findings have indicated the sympathoinhibitory effects of statins [21]. The possible molecular mechanism might involve statininduced reduction in the production of several important isoprenoids such as farnesyl pyrophosphate (FPP) and geranylgeranyl pyrophosphate (GGPP). Very recent studies have shown that statins could increase extracellular adenosine formation from adenosine monophosphate by enhancing ecto-5'-nucleotidase activity, which may result in decrease of the heart rate [22].

In the performed experiment, diltiazem significantly reduced SBP and MBP values as compared to the control group. This confirms its antihypertensive properties in the examined rabbit model and results from the direct relaxing efficacy of diltiazem associated with inhibition of the smooth muscle L-type calcium channel. In our study, simvastatin administration caused a statistically significant decrease in SBP compared to the control group. Some clinical studies have shown beneficial effects of statins on BP. The possible mechanisms responsible for the above effects have been discussed widely (Jasińska et al. 2007) and may involve an increase in NO production or a decrease in AT1 expression [23-25]. However, the BP-lowering effects of statins are observed mainly in hypertensive patients. Despite this fact, we suggest that the high dose of simvastatin used in our experiment might have an impact on the obtained results also in normolipidaemic and normotensive rabbits. The values of $\mathrm{BP}$ in rabbits receiving simvastatin and diltiazem alone or concomitantly remained at the same level. Conversely, the previous studies showed that simultaneous administration of simvastatin and another CCB, nifedipine, caused a significant decrease in $\mathrm{BP}$ values as compared to rabbits receiving nifedipine alone [17]. We suggested a mechanism based on pharmacokinetic drug-drug interaction: the suppression of nifedipine metabolism by simvastatin, resulting from competition of both drugs for CYP3A. In the present study, a further decrease in BP values after the concomitant administration of simvastatin and diltiazem was not observed. Unfortunately, due to lack of suitable equipment, in the present experiment, we were not able to measure the serum concentration of CCB to confirm the hypothesis of the pharmacokinetic drug-drug interaction at the level of isoenzyme CYP3A.

Dopamine caused a further marked reduction in TPRI in the examined groups of rabbits. Systemic and peripheral vascular resistance reduction resulting from dopamine administration is a consequence of D1 and D2 receptor stimulation [26].

The limitation of the extrapolation of our data to humans is the relatively high dose of simvastatin used in our experiments. However, the aim of the present study was to assess whether simvastatin co-administered with CYP3A inhibitor, in a dose provoking myopathy of skeletal muscles, influences myocardium efficiency. On the other hand, a much bigger role has been assigned to the aggressive lowering of LDL-C, which is connected with higher doses of hypolipaemic drugs, especially statins. As we have mentioned above, the literature findings indicated the small percentage of statin-induced musculoskeletal adverse effects (2-5\%). Thus, the number of animals used in this experiment might be insufficient. However, a high dose of simvastatin (50 mg/kg) administered to animals seems to increase the likelihood of myotoxicity incidence.

It has been suggested that changes of haemodynamic parameters might be the con- 
sequence of interaction at the isoenzyme CYP3A level. Previous findings have confirmed the functional similarity of intestinal CYP3A forms in rabbits and humans, suggesting that the rabbit is a beneficial in vivo model for the assessment of drug interaction occurring at the first pass of drugs ingested. Moreover, studies performed on rabbits, evaluating the pharmacokinetics of other drugs metabolized in humans via the CYP3A pathway (i.e. diltiazem, saquinavir) [27], have confirmed the usefulness of the rabbit model for such investigations.

In conclusion, the role of HMGRIs in the primary and secondary prevention of ischaemic heart disease is well established. However, the mechanism of the highest possible statin affinity to the skeletal muscle in comparison to the cardiac muscle in the development of HMGRI myotoxicity has not been determined. In the performed study, the concomitant administration of diltiazem, a CYP3A inhibitor, with statin surprisingly resulted in an increase of $\mathrm{Cl}$, which might suggest improved cardiac efficiency resulting from such interaction. We hypothesize that the impact of diltiazem coadministered with simvastatin on myocardium might be the consequence of both CYP3A inhibition and the beneficial influence on calcium signaling. Additionally, lack of diltiazem affinity to L-type calcium channels in myocytes may elucidate a greater risk of skeletal muscle toxicity after simvastatin and diltiazem compared to myocardium.

Changes noted in other haemodynamic parameters (BP, HR, TPRI) may throw some light on the complex impact of statin administration on cardiovascular performance, the variety of mechanisms still remaining questionable.

\section{Acknowledgments}

The study was supported by the Medical University of Lodz, grant no. 503-3011-2.

\section{References}

1. Motsko SP, Russmann S, Ming EE, et al. Effectiveness of rosuvastatin compared to other statins for the prevention of cardiovascular events-a cohort study in 395039 patients from clinical practice. Pharmacoepidemiol Drug Saf 2009; 18: 1214-22.

2. Baigent C, Keech A, Kearney PM, et al. Efficacy and safety of cholesterol-lowering treatment: prospective metaanalysis of data from 90,056 participants in 14 randomised trials of statins. Lancet 2005; 366: 1267-78.

3. Yusuf S, Lonn E, Bosch J. Lipid lowering for primary prevention. Lancet 2009; 373: 1152-5.

4. Niska R, Han B. Statins for secondary cardiovascular disease prevention for older primary care patients. J Natl Med Assoc 2009; 101: 705-10.

5. Fuentes B, Martínez-Sánchez P, Díez-Tejedor E. Lipidlowering drugs in ischemic stroke prevention and their influence on acute stroke outcome. Cerebrovasc Dis 2009; 27 Suppl 1: 126-33.

6. Ucar M, Mjorndal T, Dahlqvist R. HMG-CoA reductase inhibitors and myotoxicity. Drug Saf 2000; 22: 441-57.

7. Owczarek J, Jasińska M, Orszulak-Michalak D. Druginduced myopathies. An overview of the possible mechanisms. Pharmacol Rep 2005; 57: 23-34.

8. Molden E, Skovlund E, Braathen P. Risk management of simvastatin or atorvastatin interactions with CYP3A inhibitors. Drug Saf 2008; 31: 587-96.

9. Galatti L, Mazzaglia G, Greco A, et al. Co-prescriptions with itraconazole and fluconazole as a signal for possible risk of drug-drug interactions: a four-year analysis from Italian general practice. Pharmacoepidemiol Drug Saf 2007; 16: 422-8.

10. Gladding P, Pilmore H, Edwards C. Potentially fatal interaction between diltiazem and statins. Ann Intern Med 2004; 140: W31.

11. Wood D. Asymptomatic individuals-risk stratification in the prevention of coronary heart disease. Br Med Bull 2001; 59: 3-16.

12. Molden E, Skovlund E, Braathen P. Risk management of simvastatin or atorvastatin interactions with CYP3A4 inhibitors. Drug Saf 2008; 31: 587-96.

13. Nakahara K, Yada T, Kuriyama M, et al. Cytosolic Ca2+ increase and cell damage in L6 rat myoblasts by HMGCoA reductase inhibitors. Biochem Biophys Res Commun 1994; 202: 1579-85.

14. Yeung P, Mosher S, Pollak P. Pharmacokinetics and metabolism of diltiazem in rabbits after a single intravenous or single oral administration. Eur J Drug Metab Pharmacokinet 1991; 16: 69-74.

15. Mousa O, Brater D, Sunblad K, et al. The interaction of diltiazem with simvastatin. Clin Pharmacol Ther 2000; 67: 267-74.

16. Neuvonen PJ, Niemi M, Backman JT. Drug interactions with lipid-lowering drugs: Mechanisms and clinical relevance. Clin Pharmacol Ther 2006; 80: 565-81.

17. Jasińska M, Owczarek J, Orszulak-Michalak D. Influence of simvastatin at high dose and nifedipine on haemodynamic parameters in rabbits. Pharm Rep 2006; 58: 48-59.

18. Nakahara K, Yada T, Kuriyama M, et al. Cytosolic Ca2+ increase and cell damage in $\mathrm{L} 6$ rat myoblasts by HMGCoA reductase inhibitors. Biochem Biophys Res Commun 1994; 202: 1579-85.

19. Westerman D, Knollmann BC, Steendijk P, et al. Diltiazem treatment prevents diastolic heart failure in mice with familial hypertrophic cardiomyopathy. Eur J Heart Fail 2006; 8: 115-21.

20. Wang P, Ba ZF, Meldrum DR, Chaudry IH. Diltiazem restores cardiac output and improves renal function after hemorrhagic shock and crystalloid resuscitation. Am J Physiol; 1992; 262: H1435-40.

21. Gomes ME, Lenders JW, Bellersen L, et al. Sympathoinhibitory effect of statins in chronic heart failure. Clin Auton Res 2010; 20: 73-8.

22. Meijer $\mathrm{P}$, Wouters $\mathrm{CW}$, van den Broek $\mathrm{PH}$, et al. Upregulation of ecto-5'-nucleotidase by rosuvastatin increases the vasodilator response to ischemia. Hypertension 2010; 56: 722-7.

23. Jasińska M, Owczarek J, Orszulak-Michalak D. Statins: a new insight into their mechanisms of action and consequent pleiotropic effects. Pharmacol Rep 2007; 59: 483-99.

24. Cicero AF, Veronesi M, Prandin MG, et al. Effects of AT1 receptor and beta1 receptor blocking on blood pressure, 
peripheral hemodynamic and lipid profile in statin-treated hypertensive hypercholesterolemic patients. Fundam Clin Pharmacol 2009; 23: 583-8.

25. Li X, Wang P, Xu X, et al. Simvastatin increases the activity of endothelial nitric oxide synthase via enhancing phosphorylation. J Huazhong Univ Sci Technolog Med Sci 2009; 29: 286-90.

26. Zhao R, Fennel W, Abel F. Effects of dopamine D1 and dopamine D2 receptor agonists on coronary and peripheral hemodynamics. Eur J Pharmacol 1990; 190: 193-202.

27. Sinko P, Kunta J, Usansky H, et al. Differentiation of gut and hepatic first pass metabolism and secretion of saquinavir in ported rabbits. J Pharmacol Exp Ther 2004; 310: 359-6. 